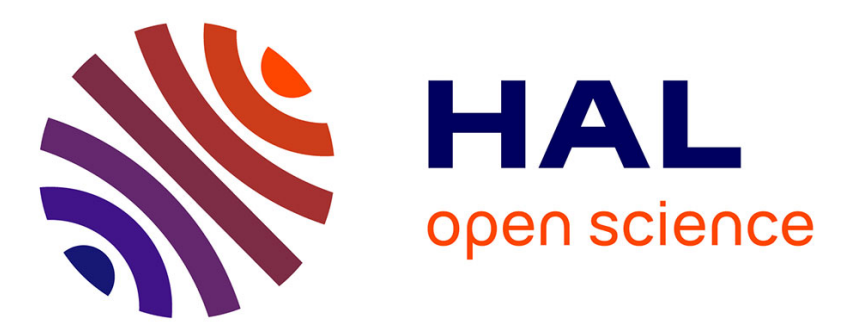

\title{
Extremely non-equilibrium oxygen plasma for direct synthesis of metal oxide nanowires on metallic substrates
}

\author{
Miran Mozetic
}

\section{To cite this version:}

Miran Mozetic. Extremely non-equilibrium oxygen plasma for direct synthesis of metal oxide nanowires on metallic substrates. Journal of Physics D: Applied Physics, 2011, 44 (17), pp.174028. 10.1088/0022-3727/44/17/174028 . hal-00613282

\section{HAL Id: hal-00613282 \\ https://hal.science/hal-00613282}

Submitted on 4 Aug 2011

HAL is a multi-disciplinary open access archive for the deposit and dissemination of scientific research documents, whether they are published or not. The documents may come from teaching and research institutions in France or abroad, or from public or private research centers.
L'archive ouverte pluridisciplinaire HAL, est destinée au dépôt et à la diffusion de documents scientifiques de niveau recherche, publiés ou non, émanant des établissements d'enseignement et de recherche français ou étrangers, des laboratoires publics ou privés. 


\title{
Extremely non-equilibrium oxygen plasma for direct synthesis of metal oxide nanowires on metallic substrates
}

\author{
Miran Mozetic
}

Department of Surface Engineering and Optoelectronics, Jozef Stefan Institute, Jamova cesta 39, SI-1000 Ljubljana, Slovenia

\begin{abstract}
A promising method for synthesis of metal oxide nanowires is based on application of extremely non-equilibrium gaseous environment found in oxygen plasma created by some types of discharges. The kinetic temperature of neutral gas is kept close to the room temperature, the electron temperature is few $\mathrm{eV}$, the ionization fraction below $10^{-6}$ and the dissociation fraction close to $100 \%$. Plasma with such characteristics is obtained using electrode-less high frequency discharges driven by radiofrequency or microwave generators. Plasma parameters such as the electron density and energy distribution function, the Debye length, the dissociation and ionization fractions, the density of negatively charged molecules, the ratio between the positively charged molecules and atoms, the distribution of atoms and molecules over excited states, etc. depend on discharge parameters. The most important discharge parameters are the generator power, frequency and coupling, the purity and pressure of working gas and the gas flow, the dimensions of the discharge chamber, the materials facing plasma, the residual atmosphere, and, usually very important though often neglected, the properties of the samples mounted into a discharge chamber. Proper construction of the experimental system for synthesis of metal oxide nanowires allows for almost $100 \%$ dissociation fraction and thus extremely rapid growing of nanowires. The particularities of oxygen plasma as well as real - time monitoring of the dissociation fraction are elaborated in this contribution. The lack of reliable experimental results on characterization of extremely non-equilibrium oxygen plasma is stressed.
\end{abstract}

1. Charged particles in cold weakly ionized plasma

Gaseous plasma is partially ionized gas. Since there are always some charged particles presented in any gas, an additional requirement to this simple definition was stated: the gas is properly called plasma if the density of charged particles is large enough to screen any DC electrical potential at a length much smaller than the typical linear dimension of plasma. For the case of non-equilibrium low pressure plasma where the electrons in unperturbed plasma have much higher kinetic energy than positive ions, the requirement is often checked by comparison of the plasma dimension with the Debye length: ionized 
gas is called plasma if the Debye length is much smaller than the plasma dimension. The Debye length is defined as

$\lambda_{D}=\sqrt{\frac{\varepsilon_{o} k T_{e}}{N_{e} e_{o}^{2}}}$

Here, $\varepsilon_{0}, \mathrm{k}, T_{e}, N_{e}$ and $\mathrm{e}_{0}$ are the vacuum permittivity, the Boltzmann constant, the electron temperature, the electron density, and the elementary charge, respectively. A typical order of magnitude for Debye length in reasonably weakly ionized plasma suitable for synthesis of metal oxide nanowires is $10^{-4} \mathrm{~m}[1-10]$. The screening effect of the space charge and the big difference between electron and ion energy in plasma cause formation of a sheath separating unperturbed plasma and any object put into plasma. There is a potential drop through the sheath. In a rough approximation the potential drop is calculated using the following equation:

$V_{p}-V_{f}=\frac{k T_{e}}{2 e_{o}} \ln \left(\frac{m_{+}}{2 m_{e}}\right)$

Here, $V_{p}$ is plasma potential, $V_{f}$ floating potential, $k$ Boltzmann constant, $T_{e}$ electron temperature, $e_{0}$ elementary charge, $m_{+}$ion mass and $m_{e}$ electron mass. The numerical value of plasma potential in oxygen is about $15 \mathrm{~V}$ for electron temperature of $k T_{e}=3 \mathrm{eV}$, and $25 \mathrm{~V}$ for electron temperature of $k T_{e}=5 \mathrm{eV}$.

The potential drop across the sheath has 2 effects: first, it rejects almost all (except very fast) electrons that would otherwise reach the surface and would be lost by surface recombination, and second, it causes acceleration of positive ions toward the surface. 
Also, the potential drop across the sheath rejects all negatively charged ions since their kinetic energy in plasma is much smaller then the voltage across the sheath.

Taken into account typical values and using equation (2) one can calculate a typical potential drop across the sheath - the order of magnitude is $10 \mathrm{~V}$. Under normal conditions, any material facing plasma is a subject to bombardment with ions of kinetic energy of the order of $10 \mathrm{eV}$. The flux of ions on the surface is, of course, limited by the flux of ions reaching the sheath, i.e.

$\mathrm{I}_{+}=1 / 4 \mathrm{e}_{0} \mathrm{n}_{+}\left\langle\mathrm{v}_{+}\right\rangle \mathrm{A}$

Here, $n_{+}$is the density of ions in unperturbed plasma, $\left\langle v_{+}\right\rangle$their average thermal velocity in plasma (in plasma created by many types of discharges it is not far from $0.04 \mathrm{eV}$ ), and A is the area. Strictly, A is the sheath area and not the area of a sample put into plasma. In the case of infinite samples, the sheath area is equal to the geometric area of a sample, and in the case of very small samples the ratio between the geometrical and sheath areas approaches 0 . In practical cases, this ratio is anywhere between 0 and 1 . In general, the sheath area is not known causing huge problems to modeling the positive ion - surface interaction for small samples.

Ion bombardment causes heating of samples that is sometimes welcome but in some other cases it is an unwanted effect. A method of avoiding ion bombardment is biasing of the sample close to the plasma potential. This is normally realized using a DC power supply. Such biasing, however, works only for small samples. Namely, biasing close to plasma potential causes huge drain of electrons from plasma (the electrons are fast in 
plasma) so the electron current to the sample should be compensated with the ion current to the counter electrode. The sheath area and/or the potential drop on the counter electrode should be increased largely to allow for compensation and this causes sputtering, what is often an unwanted effect in plasma synthesis of metal oxide nanowires since the chamber is polluted with foreign material. Still, for small samples, such avoiding of sample heating by ions work rather well providing the plasma potential is constant. Figure 1 represents a plot of a small sample temperature versus biasing. At negative biasing against plasma the sample is heated by positive ion bombardment and at positive biasing by electron bombardment. In between there is a minimum where the effect of charged particles is minimized (only neutralization energy is dissipated) and the sample is predominantly heated by other mechanisms, the main one is heterogeneous surface recombination of neutral oxygen atoms. The neutral gas temperature was about $40^{\circ} \mathrm{C}$.

Another method for avoiding heating of a sample by ion bombardment is application of plasma at moderately high pressure. In the case of collision-less sheath the ions gain the energy of $e_{0}\left(V_{P}-V_{f}\right)$ across the sheath. In an opposite case, however, they lose some energy at elastic collisions within the sheath. Since the majority of potential drop is just next to the sample, within $1 \lambda_{\mathrm{D}}$, this method for suppressing ion bombardment works at elevated pressure where the mean free path (of positive ions) is smaller than the Debye length. In practice it occurs at pressure above, say, $100 \mathrm{~Pa}$. 
In some cases, however, ion bombardment is desirous and there is a simple method for enhancing it: biasing a sample more negatively against unperturbed plasma. Obviously, best results are obtained in cases where collision-less sheath conditions are met. Strong biasing, however, should be avoided since it causes sputtering as well as secondary electron emission. The first effect causes degradation of sample and pollution of the discharge chamber with foreign material, while the other effect causes enrichment of plasma with electrons emitted from the sample. The electrons are, of course, accelerated through the sheath on the way to unperturbed plasma and cause an important enrichment if the sample is not very small.

The surface of a sample is usually far from being perfectly flat. Consequently, the real surface area of a sample is larger than the geometrical area. The roughness, however, has no effect on the sheath formation as long as the roughness (or, more precisely, the distance between two neighbor nanofeatures on a surface) is orders of magnitude smaller than the Debye length. Charged particles in unperturbed plasma therefore see a nanostructured surface as perfectly flat. The formation of the sheath has a screening effect on any nanowires that may grow on the surface of a sample as long as the wires are dense on the surface. If the sample is electrically conductive no surface charge could be accumulated at specific spots in gaps between nanowires. In the case of collision-less sheath and the distance between nanowires much smaller that the Debye length the ions' trajectories are therefore perfectly linear and perpendicular to the geometrical surface.

2. Neutral excited particles in cold weakly ionized oxygen plasma 
Understanding plasma surface interaction requires knowledge on behavior of charged particles. Plasma parameters that give basic knowledge on charged particles are the electron temperature (or electron energy distribution function, to be more precise), the density of charged particles in unperturbed plasma, the Debye length and the plasma to floating potential. The oldest and even nowadays most commonly used method for determination of these parameters is an electrical probe (often called a Langmuir probe). Electrical probes appear in several configurations including single, double and triple probes. Basically a Langmuir probe is just a piece of a metal immersed into plasma and connected to a DC power supply. The interpretation of the $\mathrm{I}=\mathrm{I}(\mathrm{U})$ characteristic, however, is often far from being trivial and requires deep understanding of many phenomena including the sheath formation, plasma oscillations, stray effects caused by EM fields, and trajectories of charged particles in the electric field.

In many (or rather most) applications of oxygen plasma, however, knowledge on charged particles is far from being sufficient since other reactive particles created in plasma often play the dominant role [11-20]. Synergistic effects between neutral reactive particles and charged particles may also play an important role. Energetic electrons in plasma cause not only ionization but also excitation of oxygen molecules and atoms. In fact, inelastic collisions that do not lead to ionization are by far more frequent in weakly ionized plasma suitable for growing metal oxide nanowires than ionization collisions. Figure 2 represents most important excited states of oxygen molecules and Figure 3 is a plot of atomic excited states 
Oxygen molecules have several excited states and most of them are metastable. The excitation energy of the first and second state is approximately 1 and $1.6 \mathrm{eV}$, respectively, and their life time is over $1 \mathrm{~s}$. Since the electron temperature is often of the order of $1 \mathrm{eV}$, it is clear that excitation of these states is much more probable that ionization. Furthermore, electron energy is easily transferred to vibrationally excited states. In oxygen, however, the vibrational temperature (or rather distribution of molecules over vibrational excited states) is always low due to a very good coupling between vibrational and translational states - supereleastic collisions between vibrationally excited molecules and oxygen atoms effectively cool the vibrational population [21].

Oxygen atoms have a variety of excited states and some are plotted in Figure 3. The first 3 excited states are metastable with the radiative decay time of 1,100 , and $0.2 \times 10^{-3} \mathrm{~s}$. The $\mathrm{O}$ atoms excited to first states are therefore pretty stable in vacuum and ready for further excitation. This is the most probable reason for observation of extremely strong emission in red part of spectrum at 777 and $845 \mathrm{~nm}$ (both lines come from excitation energy of approximately $11 \mathrm{eV}$ ).

3. Theoretical versus experimental approach

A theoretician approaching interaction between oxygen plasma and solid materials is free to choose any particle for his or her modeling. Since the life-time of normal radiative states is of the order of a ns, the theoretician will normally avoid taking into account such 
states. There is a plenty of other states that should be denser in plasma. The density of excited states depends on the production and destruction rates. While production rates have been elaborated decades ago, the destruction rates of most (meta)stable states attracted much less attention. To this end, it is difficult if not impossible to calculate the density of excited molecules and atoms in a plasma reactor. Furthermore, there are very little data on experimental determination on the density of excited states. The theoretician will thus choose particles in the ground state for his first approximation. In fact, the second approximation would be meaningful only if an experimentalist gives solid information on the density of molecules or atoms in the excited states. While there are reports on determination of the density of metastable molecular states in the literature, very little work has been done on determination of atomic excited states. The density of $\mathrm{O}$ atoms in the first excited state $\left({ }^{1} \mathrm{D}_{2}\right)$ in a laboratory plasma was measured by the group of M. Hori [22] by vacuum ultraviolet laser absorption spectroscopy. They used a microwave discharge in noble gases with small concentration of oxygen and found that the $\mathrm{O}\left({ }^{1} \mathrm{D}_{2}\right)$ density was around $10^{18} \mathrm{~m}^{-3}$. The maximum density was observed at oxygen concentration in a noble gas of about $1 \%$. They also found that the density decreases with increasing pressure. In an earlier work by Wickramanayaka, however, a much higher density of $\left({ }^{1} D_{2}\right)$ excited atoms was reported [23]. They found that the density of oxygen atoms in the first excited state exceeds $1 \times 10^{22} \mathrm{~m}^{-3}$ in an electrodeless RF plasma. Even at low discharge power of few $100 \mathrm{~W}$, the ratio between excited atoms and atoms in the ground state exceeds $50 \%$ and reaches almost $90 \%$ at $600 \mathrm{~W}$. The experiments were performed at the pressure of $133 \mathrm{~Pa}$ and the density of $\left({ }^{1} \mathrm{D}_{2}\right)$ atoms was determined by $\mathrm{H}_{2}$ titration. Their experimental procedure is explained in [24]. Most other attempts to 
measure the density of $\mathrm{O}$ atoms in the $\left({ }^{1} \mathrm{D}_{2}\right)$ excited state are limited to researchers involved in studies of upper layers of earth atmosphere. The SRI International group at Menlo Park, CA, for instance, used a powerful fluorine laser to photodissociate small amount of oxygen in helium and found about $50 \%$ atoms in the excited state [25]. The $\mathrm{O}$ $\left({ }^{1} D_{2}\right)$ density can be, of course, calculated using a suitable model. It is worth mentioning an attempt of Satu and Makabe [26] who found a pretty high ratio between atoms in excited and ground states in argon/oxygen plasma. The calculated $\mathrm{O}\left({ }^{1} \mathrm{D}_{2}\right)$ density was close to $10^{20} \mathrm{~m}^{-3}$.

A theoretician approaching interaction between oxygen plasma and solid materials should therefore wait until an experimentalist measures the density of excited $\mathrm{O}$ atoms in a chosen plasma reactor. Since the synthesis of metal oxide nanoparticles is usually performed in pure oxygen plasma, suitable experiments in pure oxygen (not a mixture of a noble gas and oxygen) are necessary. Since the potential energy of excites states is larger than the potential energy of atoms in the ground state, it is clear that they are more reactive. Unfortunately, however, the population of atoms over excited states is unknown so a theoretician approaching interaction between oxygen plasma and solid materials should stick to atoms in the ground state and any result is just the first approximation. And even determination of the neutral $\mathrm{O}$ atoms in the ground state is not trivial.

3. Oxygen atom density in a plasma reactor 
There are a handful of methods for determination the density of neutral oxygen atoms in cold weakly ionized oxygen plasma. The most commonly used are NO titration, light absorption, actinometry, mass spectroscopy and catalytic probes. They all have advantages and disadvantages so it pays to use two different methods and compare results

NO titration is a classical chemical method for determination of O density [27],[28] . It does not work properly in plasma since NO is destructed by electron impact. This method is thus usually applied for determination of the $\mathrm{O}$ density in the flowing afterglow. The value in plasma itself is then estimated using an appropriate model. Titration is based on leakage of known amount of NO (or, for safety reasons, a mixture of NO and Ar) into a flowing afterglow. The $\mathrm{NO}$ interacts with $\mathrm{O}$ to form excited $\mathrm{NO}_{2}$ molecules that are deexcited by light emission. The method is pretty reliable (although not extremely accurate) as long as back-streaming of NO into plasma is avoided.

Optical absorption techniques are becoming popular for plasma characterization [29]. They are often realized by monitoring the fluorescence of laser light absorbed by atoms. Since the radiative states suitable for application of this technique are pretty energetic at the excitation energy of about $11 \mathrm{eV}$, at least two photons should be absorbed simultaneously. In order to satisfy this condition, rather powerful lasers should be used. The laser beam is focused on the spot where $\mathrm{O}$ atom density is to be measured. The technique should be first calibrated with a noble gas (with known atom density and 
known cross-section for light quanta absorption) and then applied for oxygen. It works best in optically thin media.

Actinometry is also a popular method for estimation of the $\mathrm{O}$ atom density [33]. It is an optical emission technique based on comparison between emissions from a noble atom gas (often argon) and a suitable $\mathrm{O}$ atom line. The $\mathrm{O}$ density is then determined by comparison of intensity of an argon and an oxygen line. This method predicts a direct excitation of the oxygen atom radiative state by electron impact - in practice this assumption is usually questionable.

Mass spectrometry is a traditional method for determination of residual gas in high and ultrahigh vacuum systems. Obviously, it works well when plasma is created at high vacuum. In practice, oxygen plasma suitable for synthesis of metal oxide nanowires is usually created at elevated pressure so differential pumping is needed. [36,37]. The atoms tend to recombine on the way from plasma to the spectrometer so special care should be taken when using this method. Slits should be used instead of narrow tubes and should be made from material with a low coefficient for heterogeneous surface recombination of two atoms. The mass spectrometer should be placed as close to plasma as possible and this is often a big challenge when plasma is created by powerful RF generators. Namely, high frequency interferences may make measurements impossible and may even destroy the high impedance electrical circuits of mass spectrometers. 


\section{Catalytic probes}

Catalytic probes are the oldest method for estimation of the density of neutral atoms in plasma. As early as in 1935 Poole [39] reported application of calorimetry for estimation of the dissociation fraction. A decade later, Tollefson and Le Roy [40] used a platinum wire resistively heated to elevated temperature and they measured the power needed to sustain a constant temperature of $700 \mathrm{~K}$. They took into account an assumption of full atom recombination on the platinum wire. Much later, Trainor et al used Pt-Rd wire instead of platinum [41], but kept the assumption of full recombination. A similar apparatus was applied by Larkin [42]. Nowadays, this method is widely applied for measurements of $\mathrm{O}$ atom density in plasma created in different discharges [42-45]. The method is based on heterogeneous surface recombination of atoms. The recombination is exothermic and the dissipated energy causes heating of the solid material on whose surface recombination takes place. The power dissipated on the surface is

$$
P=j \gamma W_{O} \mathrm{~A} .
$$

Here, $j=1 / 4 n_{O}\langle v\rangle$ is the flux of atoms onto the surface, $n_{O}$ the density of atoms in plasma, $\langle\mathrm{v}\rangle$ the average thermal velocity of atoms (at room kinetic temperature of neutral gas it is $630 \mathrm{~m} / \mathrm{s}), \gamma$ is the coefficient for heterogeneous surface recombination of $\mathrm{O}$ atoms on the surface, $W_{O}$ is the average potential energy of an oxygen atom and $A$ is the surface area. The dissipated power is linear with the density of atoms in plasma. Measurements of the dissipated power should therefore give information about the $\mathrm{O}$ atom density. The technique obviously works only in the case where heterogeneous surface recombination is the major contribution of surface heating. This criterion is 
satisfied only in the case plasma is weakly ionized and not far from room kinetic temperature of neutral gas. In practice, this criterion is satisfied in plasma created by an electrodeless discharge in chambers made from material with a low recombination coefficient as long as the discharge power is reasonably low.

The advantage of a catalytic probe over other methods is straight forward: the technique is quantitative, simple and easy to use. In practice, however, there are several considerations that should be taken into account at interpretation of the results. The material that is heated should have a pretty high recombination coefficient - otherwise other mechanisms of heating may not be neglected. Next, just because of this requirement, the probe should be pretty small - otherwise it represents a solid sink of $\mathrm{O}$ atoms and thus disturbs the original density of atoms. These two requirements are rather easy to implement: the probe is made from a metal with a large recombination coefficient such as Ni or some other metals and it is made in a form of a small disc connected to tiny thermocouple wires. Such probes have, in fact, been used frequently [46-49].

A more difficult consideration is the average potential energy of $\mathrm{O}$ atoms $\left(W_{O}\right)$. A simple and not all that justified approximation is that $W_{O}$ is just a half of the dissociation energy. This approximation predicts that the majority of atoms are in the ground state. In general, this assumption is wrong since the first 3 excited states are metastable and since their excitation energy is very close to the average energy of electrons in plasma. Unfortunately, as already discussed above, little experimental work has been performed on determination of the concentration of metastable atoms in plasma. 
A catalytic probe can be heated to a very high temperature in plasma rich with $\mathrm{O}$ atoms, and may eventually melt if placed in fully dissociated plasma. This is a serious practical limitation of probe application in plasma suitable for synthesis of metal oxide nanowires. An effective way of avoiding the upper two problems is moving the probe outside the intense plasma. Basically there are at least 2 options: either to place the probe in a side arm of the discharge tube [51],[51] or in the flowing afterglow [52,53]. In principle, the probe measures the $\mathrm{O}$ density in its vicinity, so an appropriate mathematical formalism should be applied for determination of the $\mathrm{O}$ density in plasma if the probe is mounted away. Happily enough, the permeability of glass tubes for $\mathrm{O}$ atoms can be determined experimentally [54]. Figure 4 represents schematic of the experimental setup for determination of the $\mathrm{O}$ atom density in a side arm, while Figure 5 corresponds to the flowing afterglow case. In both cases, the behavior of $\mathrm{O}$ density along the tubes can be also predicted theoretically using an appropriate model providing the recombination coefficient of the tube surface is known [55].

Highly catalytic material causes a drain of oxygen atoms. The practical consequence of this fact is formation of a sheath around the catalytic tip of the probe. Obviously, there is a gradient of atoms within the sheath: the atom density just next to the catalytic material is lower than the value far away. This effect can be minimized by making the probe very small, but it always persists. An attempt has been done to measure the drain of atoms by a probe in a simple experiment with two probes [57]. One was fixed and the other was made movable. Measuring the temperature of the fixed probe versus the position of the movable probe allows for estimation of the drain. 
Another consideration that is often neglected is the local increase of the gas kinetic temperature due to gas heating by accommodation of gaseous atoms and molecules on the hot surface of the catalyst. This effect, again, can be minimized using a very small catalyst.

Yet another consideration that should be taken into account at interpretation of the probe signal is the probability for recombination. It is usually expressed in the terms of the recombination coefficient. Early authors simplified their live (and underestimated the atom density) taking into account the value 1 . Nowadays it is generally accepted that the coefficient depends on the type of catalyst material and was measured for several materials by several authors. The discrepancy between the results, however, is far from being negligible. A part of the uncertainty arises from uncertainty of the measuring method. This sort of uncertainty can be estimated. Another source, however, is the surface finish - nowadays it is known that rough materials have higher recombination coefficient than smooth materials [57],[58]. Yet the third source may be a temperature dependence of the recombination coefficient. Some materials tend to have different recombination coefficients at different temperature. As for example, the recombination coefficient for some ceramics gradually increases with increasing temperature [60],[61]. For some other materials, the recombination coefficient may jump at certain temperature. This is, for instance, the case for platinum. Figure 6 is a plot of platinum catalyst versus time at different power of the discharge. At low power (and low $\mathrm{O}$ density in the vicinity of the probe) the catalyst temperature stabilizes at a rather low value. As power is increased, the probe temperature does not rich a constant value but keeps increasing with time until, at about $750 \mathrm{~K}$, a dramatic rise of the temperature is observed. At even higher 
power, this effect is even more pronounced. Obviously, the recombination coefficient jumps at about $750 \mathrm{~K}$.

A nightmare of experimentalists using plasma created in radiofrequency discharges is electromagnetic interferences or stray effects. Radiofrequency antennas have been known for a century as effective radiation sources. An antenna acts as a transmitter and any metallic object is a receiver. In plasma application it means that any metallic part placed close to RF plasma source acts as a receiver. Some high impedance sources may actually be destroyed by this effect. From this point of view, catalytic probes should not be made from metallic components. Of course, the catalyst should be metallic in order to assure a high recombination coefficient, but the probe signal should not be transferred to an appropriate detector through metallic wires. Thermocouple probes, in fact, work only far from RF coils. A suitable solution to overcome electromagnetic interferences is introduction of fibre optics catalytic probes (FOCP) a decade ago [62]. Schematic of the FOCP is presented in Figure 7. The catalyst is connected to an optical fibre. The increased probe temperature due to heterogeneous surface recombination causes radiation in the IR or visible range and this radiation is lead through the optical fibre to a suitable optoelectronic detector. Since the connection is optical no interferences reach detector (which is mounted into a Faraday cage) so this problem is solved.

A typical example of the $\mathrm{O}$ atom density measured with a FOCP is presented in Figure 8. The $\mathrm{O}$ atom density increases with pressure at rather low pressure, reaches a maximum and decreases with further pressure increase. This is a typical behavior and was observed 
in numerous systems. The appearance of the maximum is explained by 2 basic facts: first, at low pressure, the electron temperature is rather high and so is the dissociation probability. Second, at high pressure, the electron temperature becomes small and so does the electron density so the dissociation frequency is rather low. Also, at high pressure, the loss of atoms by gas phase recombination becomes important as the probability for threebody collisions increases with increasing pressure.

The electrons readily dissociate oxygen molecules at low pressure. The production of atoms is thus limited just by the supply of molecules. The loss of atoms, on the other hand, practically does not depend on gas phase phenomena, but rather on surface phenomena - heterogeneous surface recombination. Not surprisingly, the density of $\mathrm{O}$ atoms at low pressure depends on the type of materials facing plasma. In metallic chambers, the surface recombination coefficient is orders of magnitude larger than in glass chambers. In fact, it was recently found that the $\mathrm{O}$ atom density in a metallic chamber is 2 orders of magnitude smaller than in glass chambers, although the discharge parameters are almost identical [63]. This fact has a serious consequence in synthesis of metal oxide nanowires: the $\mathrm{O}$ atom density depends on the properties of samples immersed into a plasma reactor, as shown recently [64]. The effect of $\mathrm{O}$ atom drain by the samples exposed to oxygen plasma afterglow is even more pronounced. In fact, the $\mathrm{O}$ atom density may fall below the detection limit of a probe if the interaction between a sample and the $\mathrm{O}$ atoms is intensive [65]. 
From the upper considerations it is clear that the $\mathrm{O}$ atom density in a reactor depends not only on the properties of the sample to be treated but also on the history of a sample - the $\mathrm{O}$ atom density may therefore change with time depending on the surface conditions of a sample, and this change is observed although the discharge parameters are kept perfectly constant $[66,67,68]$

All upper considerations should be taken into account when attempting to develop a model of nanowire growth during exposure of metal foils to oxygen plasma. The first approximation (i.e. taking into account only atoms in the ground state) is probably insufficient due to the simple fact that nanowires usually do not grow in moderately nonequilibrium plasma. Extremely non-equilibrium plasma should be used in order to provide the critical flux of atoms (probably also those in excited states) that enables rapid synthesis of one dimensional crystals of metal oxide. Figure 9 represents SEM images of same material (niobium foil) exposed to moderately and extremely non-equilibrium plasma. In the first case the oxide grains are similar to those growing at thermal oxidation, but in the case of extremely non equilibrium plasma nice bundles of nanowires are formed during plasma treatment. Here, is should be stressed that the exact mechanism of the nanowire growth is still not known. Although some attempts $[7,12,70]$ have been done to enlighten the phenomenon, a complete picture of the growth mechanism and the influence of different plasma particles on the nanowire properties is to be elaborated in the next future.

\section{Outlook}


Weakly ionized highly dissociated oxygen plasma has been successfully applied for synthesis of metal oxide nanowires. The authors showed that the phenomenon depends on plasma parameters and generally agree that the major reactants are neutral oxygen atoms. The density of $\mathrm{O}$ atoms is determined by few techniques, and catalytic probes performed particularly well. The exact model of growing mechanism, however, is not yet known. In particular, no data is available on interaction of excited oxygen atoms with substrates. Although some attempts have been done to determine the density of $\mathrm{O}$ atoms in the first excited state, the results cannot be generalized, and they also vary for orders of magnitude. It seems that the results depend largely on the experimental technique applied by particular authors. Obviously, such results cannot be applied for modeling of the plasma -surface interaction let alone practical applications. Therefore, there is a need for i) measurements of $\mathrm{O}\left({ }^{1} \mathrm{D}_{2}\right)$ in different plasma reactors and by different techniques, and ii) development of techniques suitable for determination of the density of $\mathrm{O}$ atoms in other most important metastable excited states. In fact, many techniques available nowadays can be adopted for measurements of oxygen atom excited states but the authors that may attempt to use them should estimate critically the accuracy of the applied technique. And, as usual, they are advised to use at least 2 different techniques simultaneously - only comparison of results at exactly the same experimental conditions can give valuable data on practical application of different experimental techniques. Collaboration of experts specialized in specific technique is encouraged. Some attempt has been already realized and they include comparison of TALIF and catalytic probes [69]. 
Reliable results on determination of the density of oxygen atoms in excited states will be very useful for i) improvement of the accuracy of catalytic probes, and ii) development of a suitable model of metal - oxygen plasma interaction that leads to spontaneous growth of metal oxide nanowires on the surface of metal samples during treatment with extremely non-equilibrium oxygen plasma.

\section{References}

[1] Mozetic M, Cvelbar U, Sunkara KM and Vaddiraju S 2005 Advan. Mater. 17 21382142

[2] Cvelbar U, Chen ZQ, Sunkara MK and Mozetic M 2008 Small 4 1610-1614

[3] Cvelbar U, Ostrikov K, Levchenko I, Mozetic M and Sunkara MK 2009 Appl. Phys. Lett. 94211502

[4] Ostrikov K and Murphy AB 2007 J. Phys.D.: Appl. Phys. 40 2223-2241

[5] Mariotti D, Bose AC and Ostrikov K 2009 IEEE Trans. Plasma Sci. 37 1027-1033

[6] Mariotti D, Lindstrom H, Bose AC and Ostrikov K 2008 Nanotechnology 19 495302

[7] Cvelbar U and Ostrikov 2008 Cryst. Growth Des. 40 1444-1453

[8] Rao R, Chandrasekaran H, Gubbala S, Sunkara MK, Daraio C, Jin S and Rao AM 2006 J. Electron. Mater. 35 941-926

[9] Kumar V, Kim J H, Pendyala C, Chernomordik and Sunkara MK 2008 J. Phys. Chem. C 112 17750-17754 
[10] Kim J H, Kumar V, Chernomordik B and Sunkara M K 2008 Inform. MIDEM 4 237-243.

[11] Junkar I, Cvelbar U, Vesel A, Hauptman N and Mozetic M 2009 Plasma Processes Polym. 6 667-675

[12] Cvelbar U, Ostrikov K and Mozetic M 2008 Nanotechnology19 405605

[13] Vesel A, Junkar I, Cvelbar U, Kovac J and Mozetic M 2008 Surf. Interface Anal. 40 1444-1453

[14] Vesel A, Mozetic M and Zalar A 2002 Appl. Surf. Sci. 200 94-103

[15] Vesel A, Mozetic M, Drenik A, Hauptman N and Balat-Pichelin M 2008 Appl. Surf. Sci. 255 1759-1765

[16] Vesel A, Mozetic M, Hladnik A, Dolenc J, Zule J, Milosevic S, Krstulovic N, Klanjsek-Gunde M and Hauptman N 2007 J. Phys. D: Appl. Phys. 40 3689-3696

[17] Krstulovic N, Labazan I, Milosevic S, Cvelbar U, Vesel A and Mozetic M $2006 \mathrm{~J}$. Phys. D: Appl. Phys. 39 3799-3804

[18] Vesel A, Mozetic M, Drenik A, Milosevic S, Krstulovic N, Balat-Pichelin M, Poberaj I anda Babic D 2006 Plasma Chem. Plasma P. 26 577-584

[19] Levchenko I, Cvelbar U and Ostrikov K 2009 Appl. Phys. Lett. 95021502

[20] Mafra M, Belmonte T, Poncin-Eppaillard F and Maliska A 2009 Plasma Processes Polym. 6 S198-S203

[21] Ricard A 1996 Reactive plasmas SFV Societe Francaise du Vide

[22] Takeda K, Takashima S, Ito M and Hori M 2008 Appl. Phys. Lett. 93021501

[23] Wickramanayaka S, Hosokawa N and Hatanaka Y 1991 Jap. J. Appl. Phys. 30 2897-2900 
[24] Wickramanayaka S, Mekile S, Sekiguchi S, Hosokawa N and Hatanaka Y $1991 \mathrm{~J}$. Appl. Phys. 691

[25] Kalogerakis K S, Slanger T G, Kendall E A, Pedersen T R, Kosch M J, Gustavsson B and Rietveld M T 2009 Ann. Geophys., 27 2183-2189

[26] Satu T and Makabe T 2008 J. Phys. D: Appl. Phys. 41035211

[27] Mozetic M, Ricard A, Babic D, Poberaj I, Levaton J, Monna V and Cvelbar U 2003 J. Vac. Sci. Technol. A 21 369-374

[28] Belmonte T, Pintassilgo C D, Czerwiec T, Henrion G, Hody V, Thiebaut J M and Loureiro J 2005 Surf. Coat. Technol. 200 26-30

[29] Stancu G D, Kaddouri F, Lacoste D A and Laux C O 2010 J. Phys. D: Appl. Phys. 43124002

[30] Hayashi Y, Hirao S, Zhang Y, Gans T, O'Connell D, Petrovic Z Lj and Makabe T 2009 J. Phys. D 42145206

[31] Cardoso RP, Belmonte T, Henrion G and Sadeghi N 2006 J. Phys. D: Appl. Phys. 39 4178-4185

[32] Krstulovic N, Cutic N and Milosevic S 2009 Spectrochem. Acta B 64 271-277

[33] Balat-Pichelin M, Passarelli M and Vesel A 2010 Mater. Chem. Phys. 123 40-46

[34] Balat-Pichelin M and Beche E 2010 Appl. Surf. Sci. 256 4906-4914

[35] Nagai H, Hiramatsu M, Hori M and Goto T 2003 Rev. Sci. Instrum. 74 3453-3459

[36] Czerwiec T, Gavillet J, Belmonte T, Michel H and Ricard A 1998 Surf. Coat. Technol. 98 1411-1415

[37] Ferreira JA, Tabares FL and Tafalla D 2009 J. Nucl. Mater. 390-391 592-596

[38] Ferreira JA, Tabares FL 2007 J. Vac. Sci. Technol. A 25 246-251 
[39] Poole H G 1937 Proc. Roy. Soc. A 163415

[40] Tollefson E L and Le Roy D J 1948 J. Chem. Phys. 581057

[41] Trainor D W, Ham D O and Kaufman F 1973 J. Chem. Phys. 584599

[42] Larkin F S 1967 Can. J. Chem. 461005

[43] Balat-Pichelin M and Vesel A 2006 Chem. Phys. 327 112-118

[44] Vesel A, Mozetic M and Balat-Pichelin M 2007 Vacuum 81 1088-1093

[45] Mozetic M, Vesel A, Cvelbar U and Ricard A 2006 Plasma Chem. Plasma P. 26 $103-117$

[46] Mozetic M, Vesel A, Monna V and Ricard A 2003 Vacuum 71 201-205

[47] Ricard A, Gaillard M, Monna V, Vesel A and Mozetic M 2001 Surf. Coat. Techol. 142-144 333-336

[48] Drenik A, Cvelbar U, Ostrikov K and Mozetic M 2008 J. Phys. D: Appl. Phys. 41 115201

[49] Mozetic M, Cvelbar U, Vesel A, Ricard A, Babic D and Poberaj I 2005 J. Appl. Phys. 97 103308-1-103308-7

[50] Mozetic M, Vesel A, Drenik A, Poberaj I and Babic D 2007 J. Nucl. Mater. 363$3651457-1460$

[51] Drenik A, Cvelbar U, Vesel A and Mozetic M 2005 Inf. Midem 35 85-91

[52] Cvelbar U, Ostrikov K, Drenik A and Mozetic M 2008 Appl. Phys. Lett. 92133505

[53] Cvelbar U and Mozetic M 2007 J. Phys.D: Appl. Phys. 402300

[54] Cvelbar U, Mozetic M, Babic D, Poberaj I and Ricard A 2006 Vacuum 80904

[55] Sorli I and Rocak R 2000 J. Vac. Sci. Technol. A. 18 338-342 
[56] Drenik A 2009 Probability of heterogeneous recombination of hydrogen and oxygen atoms on the surfaces of fusion-relevant materials, PhD Thesis, Jozef Stefan Int. Postgradual School Ljubljana

[57] Mozetic M 1997 Interaction of hydrogen plasma with solid surfaces, PhD Thesis, University of Maribor

[58] Drenik A, Vesel A and Mozetic M 2009 J. Nucl. Mater. 386-388 893-895

[59] Drenik A, Tomeljak A, Mozetic M, Vesel A, Babič D and Balat-Pichelin M 2010 Vacuum 84 90-93

[60] Balat-Pichelin M, Hernandez D, Olalde G, Rivoire B and Robert J F 2002 J. Sol. Energ.-T. ASME 124 15-222

[61] Balat-Pichelin M, Badie J M, Berjoan R and Boubert P 2003 Chem. Phys. 291 181194

[62] Babic D, Poberaj I and Mozetic M 2001 Rev. Sci. Instrum. 72 4110-4114

[63] Vrlinic T, Mille C, Debarnot D and Poncin-Eppaillard F 2009 Vacuum 83 792-796

[64] Mozetic M and Cvelbar U 2009 Plasma Sources Sci. Technol. 18034002

[65] C. Canal, Gaboriau F, Ricard A, Mozetic M, Cvelbar U and Drenik A 2007 Plasma Chem. Plasma Process. 27 404-413

[66] Junkar I, Cvelbar U, Vesel A, Hauptman N and Mozetic M 2009 Plasma Process. Polym. 6 667-675

[67] Canal C, Gaboriau F, Villeger S 2009 Int. J. Pharm. 367 155-161

[68] Vesel A and Mozetic M 2001 Vacuum 61 373-377

[69] Gaboriau F, Cvelbar U, Mozetic M, Erradi and Roufflet B 2009 J. Phys. D: Appl. Phys. 42055204. 
[70] Ostrykov K, Levicenko I, Cvelbar U, Sunkara M and Mozetic M 2010 Nanoscale, in press. 


\section{FIGURE CAPTIONS}

Figure 1. Sample temperature versus bias. The rather broad minimum is obtained due to finite electron temperature and probably also due to oscillations of plasma to sample potential.

Figure 2. Some excited states of oxygen molecules.

Figure 3. Some excited states of oxygen atoms. The approximate radiative life time of some metastable states are indicated.

Figure 4. An Experimental system for determination of the $\mathrm{O}$ atom density in a side arm of the discharge tube and a relative density of $\mathrm{O}$ atoms versus the depth.

Figure 5. The $\mathrm{O}$ atom density along a continuously pumped glass tube.

Figure 6. The temperature of platinum catalyst at different MW discharge powers (and thus different density of $\mathrm{O}$ atoms) in the flowing afterglow.

Figure 7. Schematic of a fibre optics catalytic probe. 
Figure 8. Typical behavior of the $\mathrm{O}$ atom density in a $\mathrm{RF}$ plasma reactor. The discharge chamber is a long Pyrex cylinder with diameter of $4 \mathrm{~cm}$, and the useful discharge power is about $100 \mathrm{~W}$.

Figure 9. SEM images of niobium foil exposed to moderately (a) and extremely (b) nonequilibrium oxygen plasma. The width of each image is $6 \mu \mathrm{m}$. Both images were taken sample tilting of $45^{\circ}$.

Boxed text 


\section{BOXED TEXT}

Gases in thermal equilibrium are described by 3 simple equations:

$$
\begin{aligned}
& p V=\frac{m}{M} R T_{1} \\
& \frac{N_{A}}{2 N_{M}}=e^{-W_{D} / k T_{2}} \\
& \frac{N_{I}}{N_{M}}=e^{-W_{i}} / k T_{3}
\end{aligned}
$$

$\mathrm{P}$ is the gas pressure, $\mathrm{V}$ its volume, $\mathrm{m}$ the molecule mass, $\mathrm{M}$ the kilomole mass, $\mathrm{R}$ the gas constant $\left(8.314 \mathrm{~J} \cdot \mathrm{K}^{-1} \cdot \mathrm{mol}^{-1}\right)$ and $\mathrm{T}_{1}$ the gas kinetic temperature (i.e. a measure of the molecule average kinetic energy). $N_{A} / 2 N_{M}$ is the dissociation fraction of oxygen molecules, $\mathrm{W}_{\mathrm{D}}$ the dissociation energy, $\mathrm{k}$ Boltzmann constant, $\mathrm{T}_{2}$ the dissociation temperature (i.e. a measure of the dissociation fraction), $\mathrm{N}_{\mathrm{I}} / \mathrm{N}_{\mathrm{M}}$ the ionization fraction of oxygen molecules, and $\mathrm{T}_{3}$ the ionization temperature (i.e. a measure of the ionization fraction. In principle, $T_{1}$ has nothing in common with $T_{2}$ or $T_{3}$. For gases in thermodynamic equilibrium

$T_{1}=T_{2}=T_{3}=T$

and all the T's are simply called "gas temperature". In nonequilibrium state of gas, however, the relation (4) is not satisfied. Furthermore, even the kinetic temperature $\left(\mathrm{T}_{1}\right)$ may be different for different particles found in gas (neutral molecules, ions, electrons). In some cases, the distribution of particles over translational, rotational, vibrational and/or electronically excited states does not obey Maxwell - Boltzmann law so the state of gas cannot be described by equations (1-3). Gas is therefore away from thermodynamic equilibrium if any of upper requirements are not satisfied. In a typical low-pressure oxygen plasma the kinetic temperature of neutral gas is often not much above the room temperature (i.e. $\mathrm{T}_{\mathrm{N}}=300 \mathrm{~K}$ ), the electron temperature is often 2 orders of magnitude larger (i.e. at around $\mathrm{T}_{\mathrm{e}}=30,000 \mathrm{~K}$ ), while the positive ion kinetic temperature is anywhere between $T_{N}$ and $T_{e}$ (in weakly ionized plasma often close to $\mathrm{T}_{\mathrm{N}}$ ). The dissociation and ionization temperatures are often of the same order of magnitude as $\mathrm{T}_{\mathrm{e}}$. Plasma is often called "extremely nonequilibrium" if huge differences in temperatures are observed. For instance, if the neutral gas kinetic temperature is $300 \mathrm{~K}$, while either $\mathrm{T}_{2}$ or $\mathrm{T}_{3}$ is over $10^{5}$ $\mathrm{K}$. In practice it is difficult to achieve high ionization fraction of molecules at low kinetic temperature, so a very typical extremely non-equilibrium oxygen plasma would have the dissociation temperature orders of magnitude larger than the neutral gas temperature. As for en example, $\mathrm{T}_{2}$ is about $6 \times 10^{5} \mathrm{~K}$ at the dissociation fraction of $90 \%$, and $6 \times 10^{6} \mathrm{~K}$ at dissociation fraction of $99 \%$. 


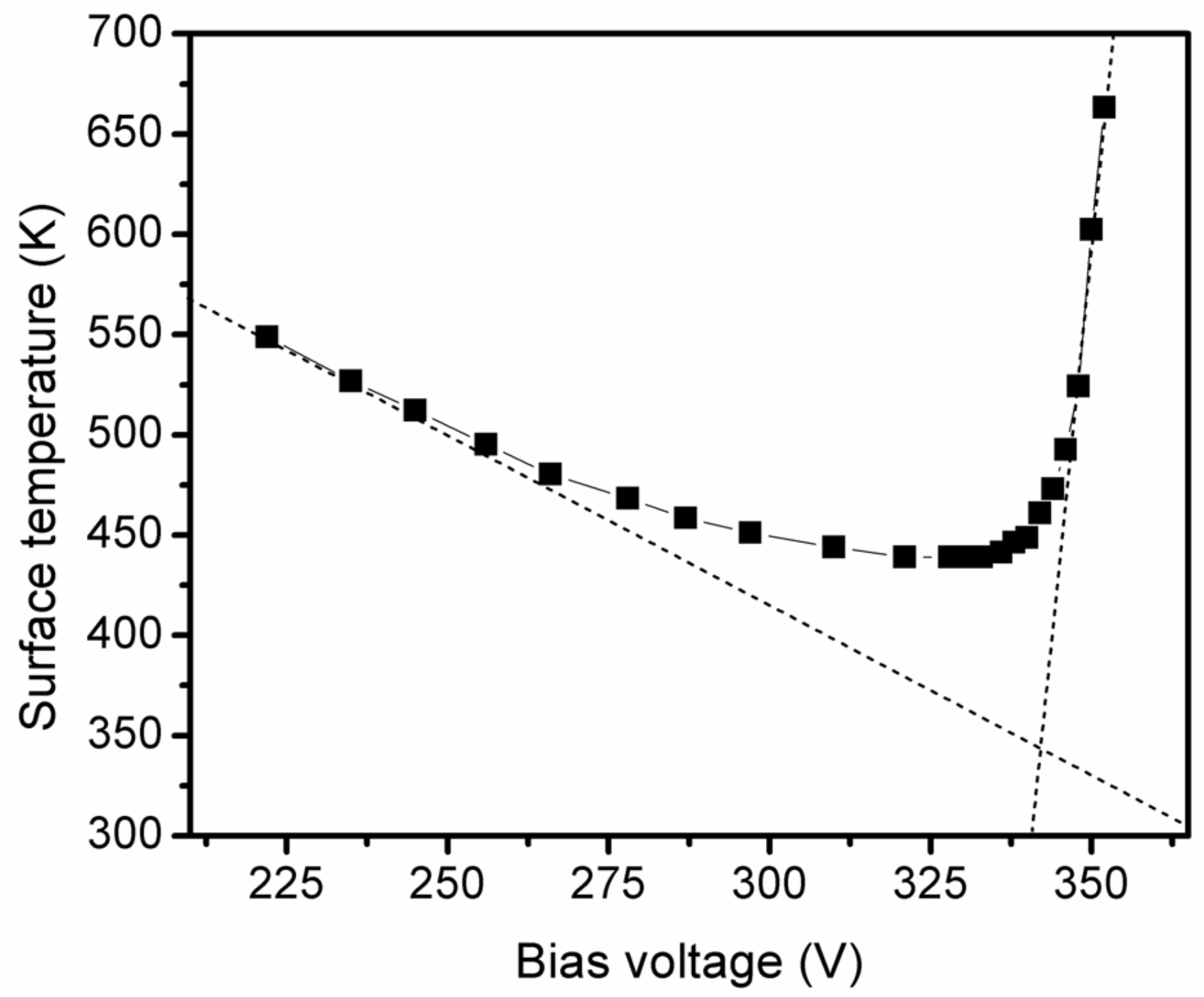

Figure 1 (Figure1.tif) 


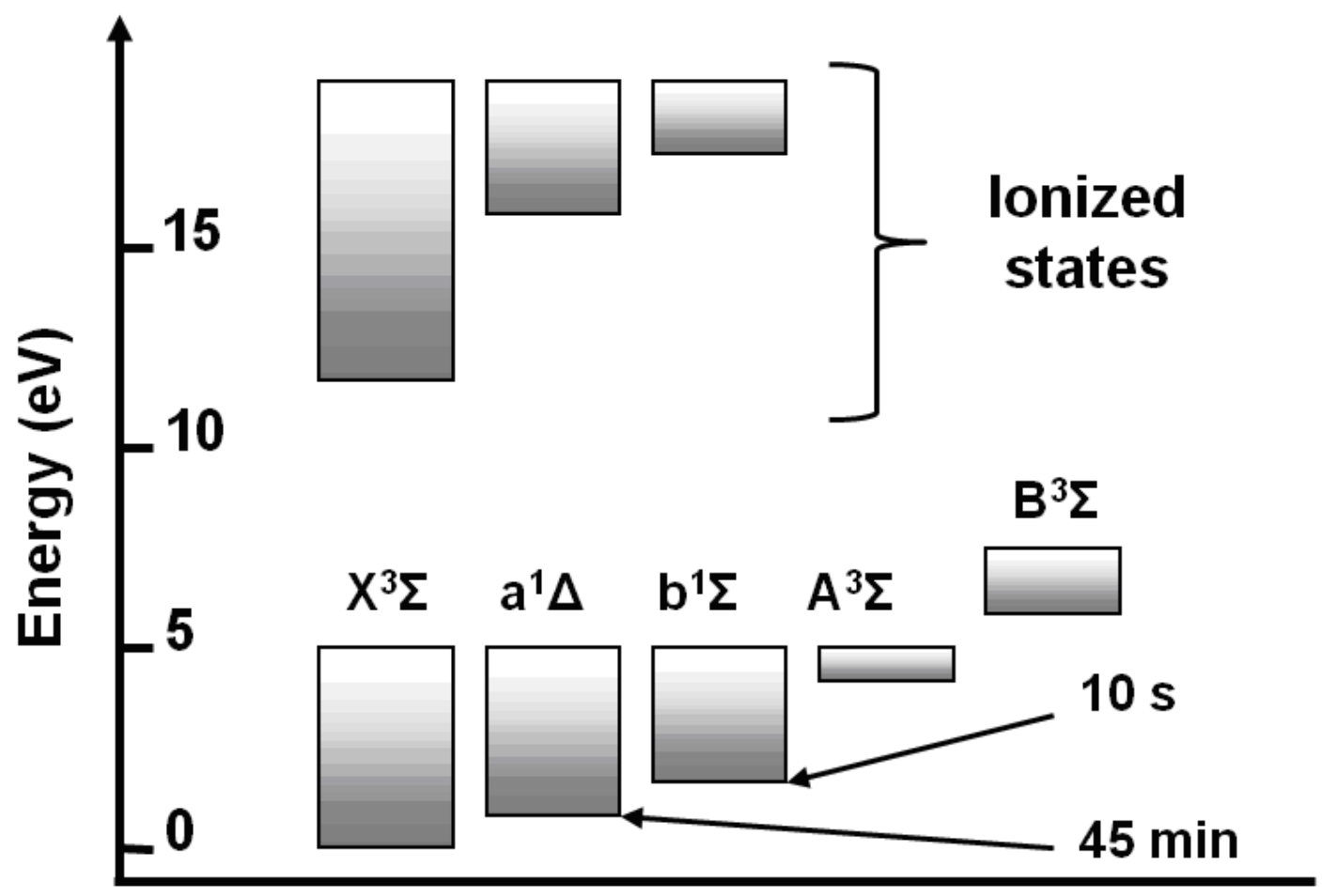

Figure 2 (Figure2.tif) 


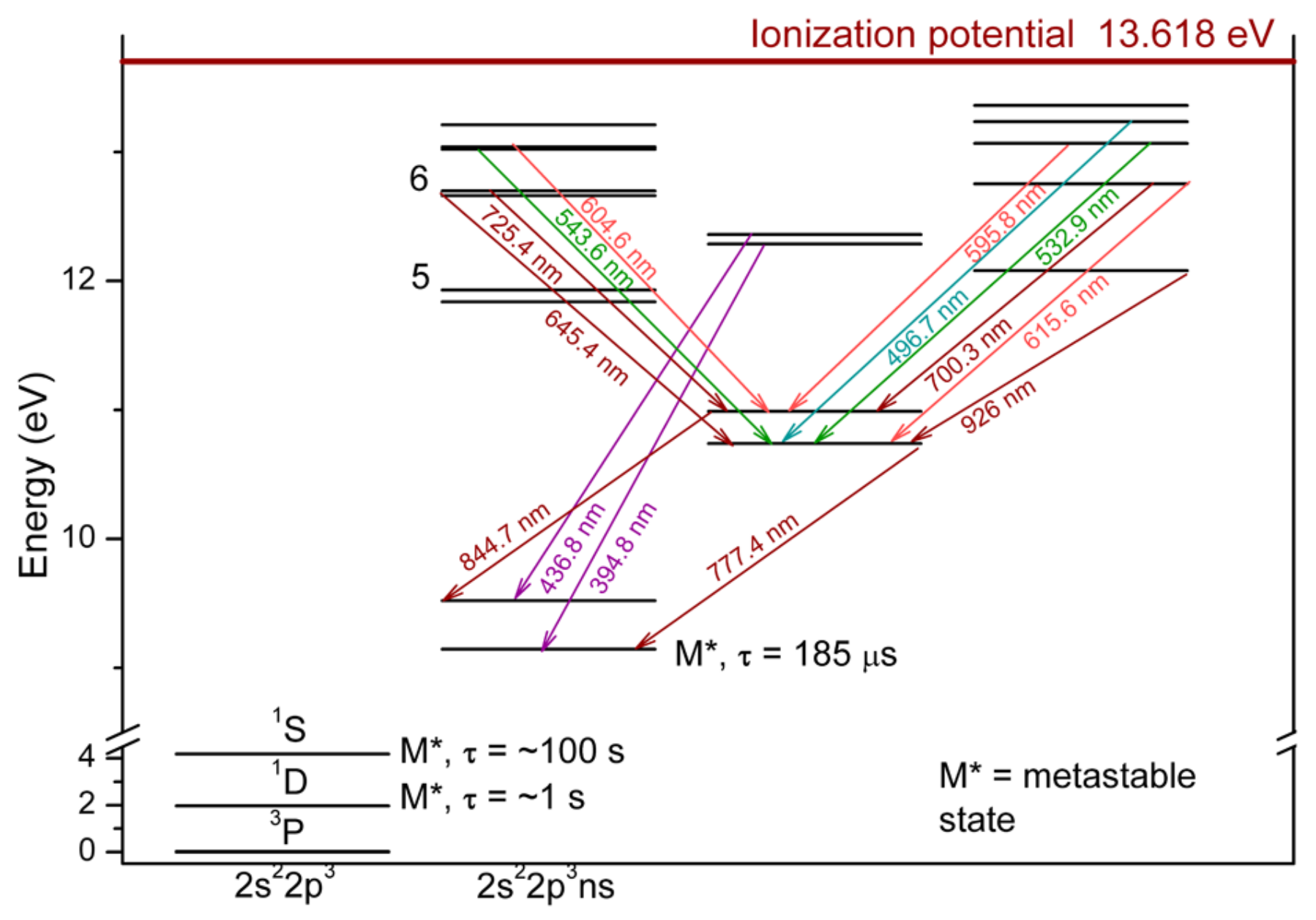

Figure 3 (Figure3.tif) 


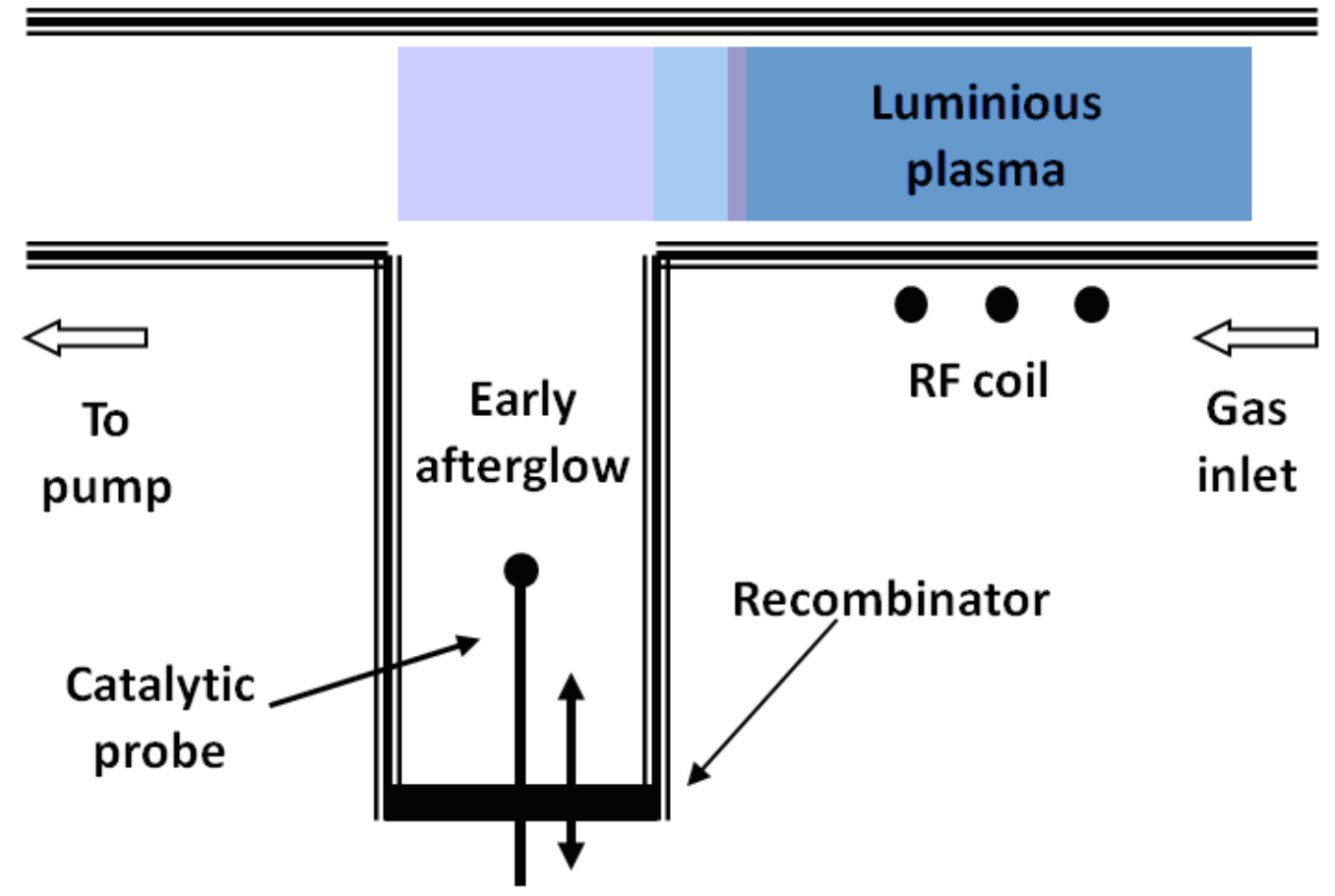

Figure 4 (Figure4.tif) 


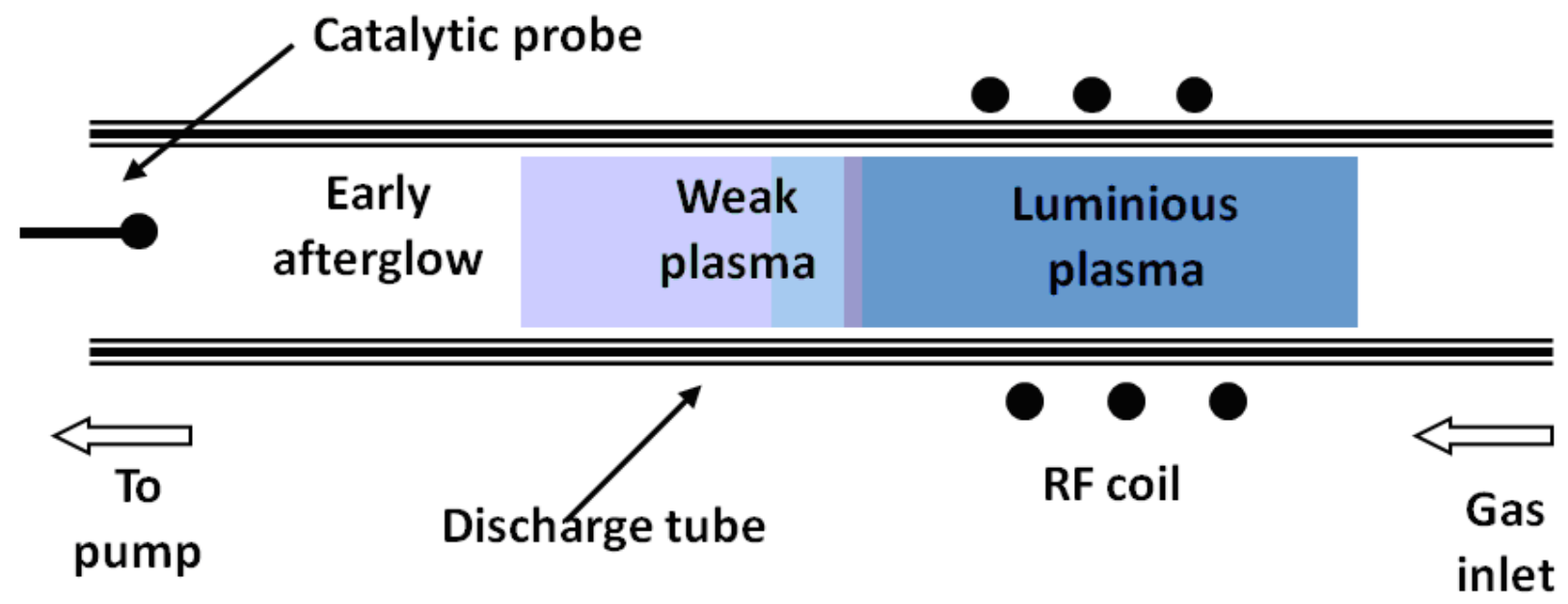

Figure 5 (Figure5.tif) 


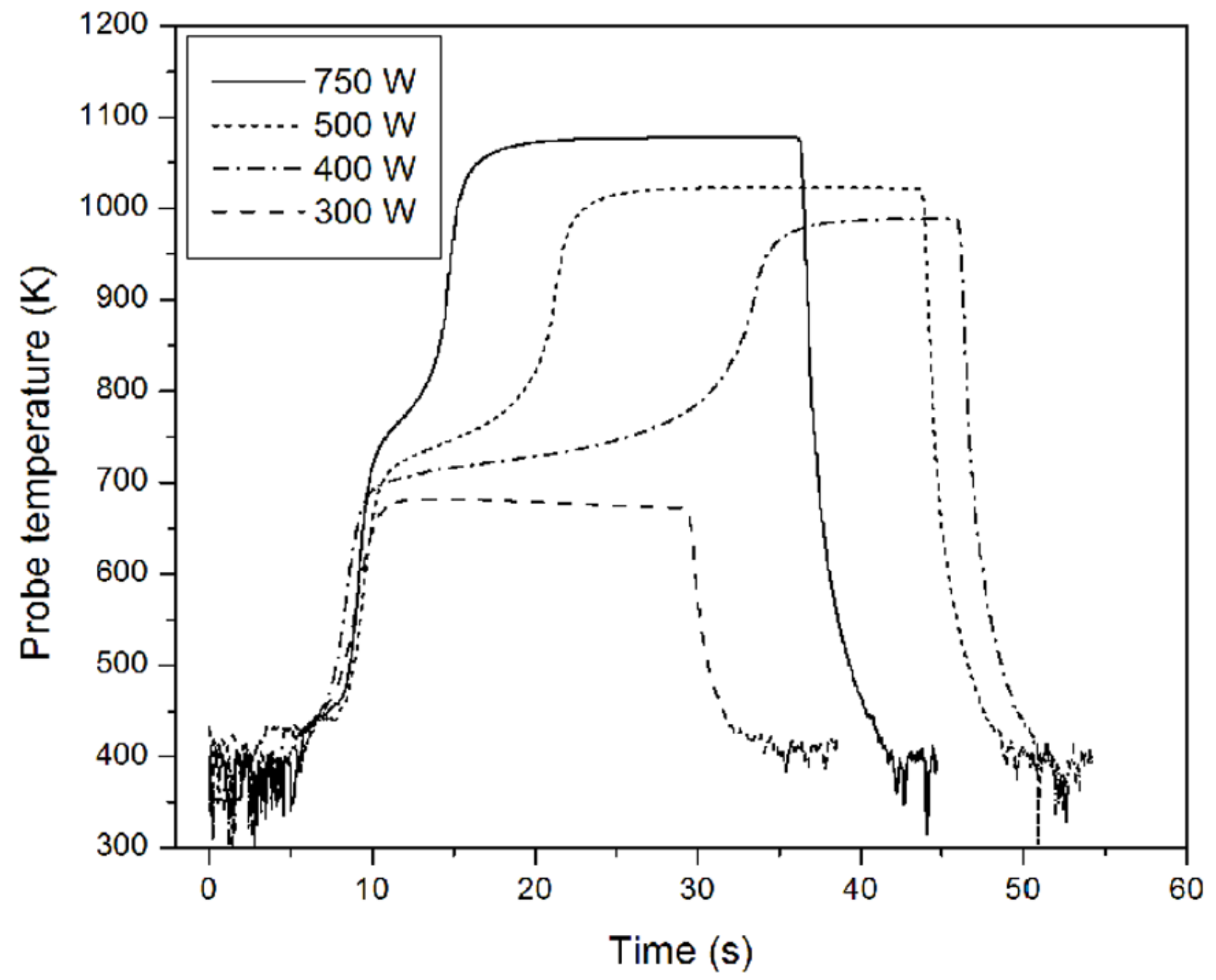

Figure 6 (Figure6.TIF) 
Catalytic material

housing

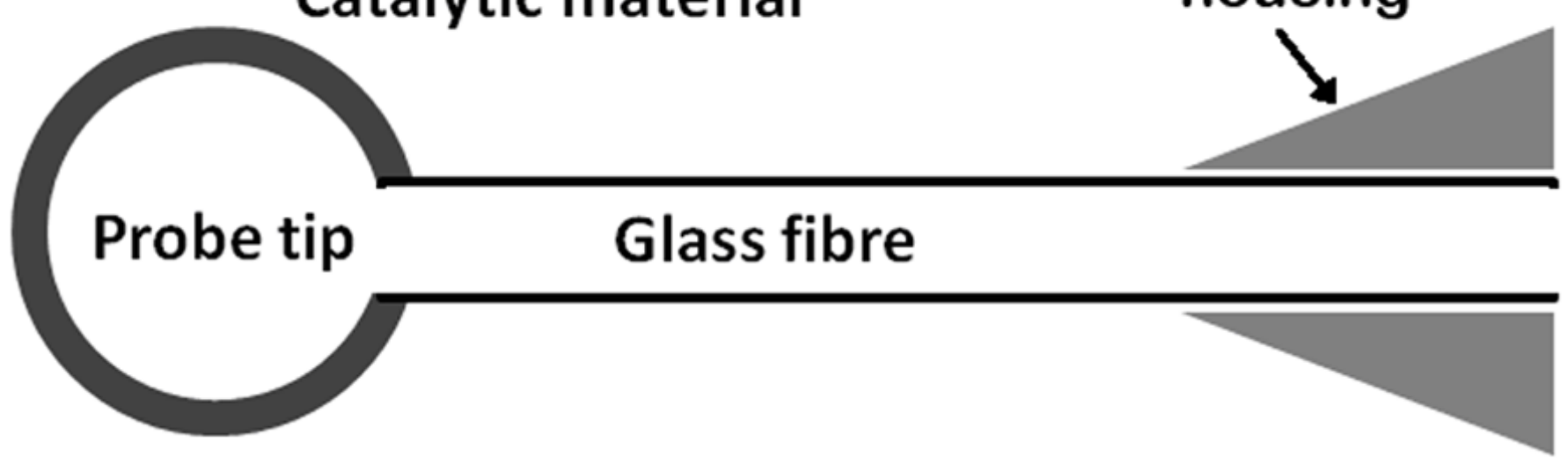

Figure 7 (Figure7.tif) 


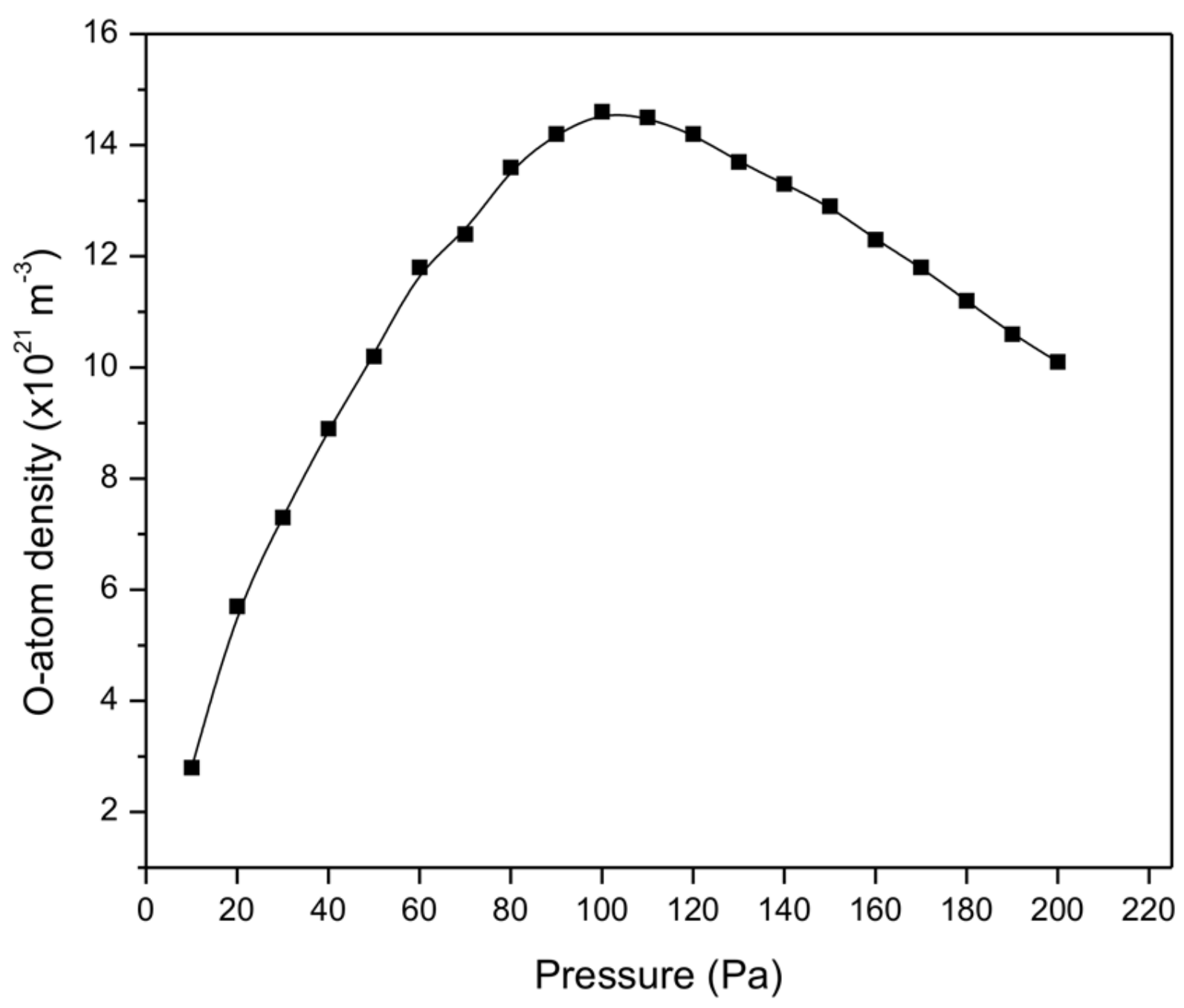

Figure 8 (Figure8.tif) 


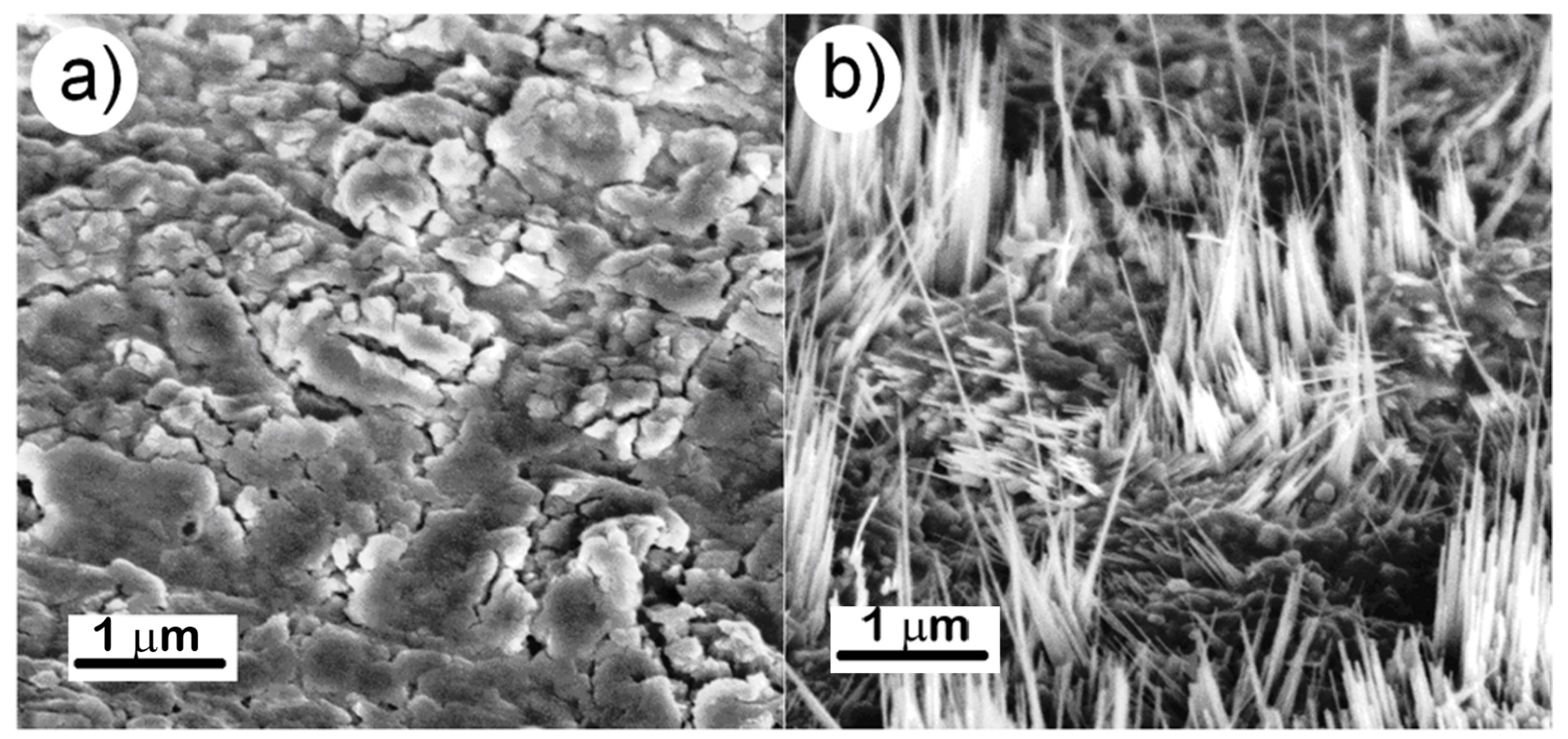

Figure 9 (Figure9.jpg) 